\title{
Nalmefene, Given as Needed, in the Routine Treatment of Patients with Alcohol Dependence: An Interventional, Open-Label Study in Primary Care
}

\author{
Philippe Castera ${ }^{a}$ Edmund Stewart ${ }^{b}$ Josef Großkopf ${ }^{c}$ Carlos Brotons $^{d}$ \\ Maiken Brix Schou ${ }^{e}$ Doris Zhang ${ }^{e}$ Björn Steiniger Brach ${ }^{f}$ \\ Didier Meulien $^{\mathrm{e}}$ on behalf of the PICASO Study Investigators \\ a Professeur Associé de Médecine Générale De I'Université De Bordeaux, Cabinet Medical, Bordeaux, France; \\ ${ }^{\mathrm{b}} \mathrm{NHS}$ Lanarkshire Integrated Addiction Service, Coathill, Lanarkshire, UK; ${ }^{\mathrm{C}}$ Gemeinschaftspraxis, Innere und \\ Allgemeinmedizin, Wallerfing, Germany; ${ }^{d}$ Sardenya Primary Health Care Center, Biomedical Research Institute \\ Sant PauPau (IIB-Sant Pau), Barcelona, Spain; ${ }^{\mathrm{e}} \mathrm{H}$. Lundbeck A/S, Valby, Denmark; ${ }^{\mathrm{f} U C B}$ Biopharma S.P.R.L, Brussels, \\ Belgium
}

\section{Keywords}

Alcohol dependence · Primary care · Nalmefene ·

Clinical trial

\begin{abstract}
Aims: This 12-week, open-label, primary care study (NCT02195817) evaluated the efficacy and safety of naImefene, taken as needed, to reduce alcohol consumption in adults with a diagnosis of alcohol dependence and drinking at least at high drinking risk levels (DRL, >60 g/day for men, $>40 \mathrm{~g}$ /day for women). Methods: Following the Screening Visit, patients recorded their daily alcohol consumption for 2 weeks. Patients were then categorised by their self-reported drinking levels; those who maintained at least a high DRL in the 2-week period were included in Cohort-A, and those who reduced their alcohol consumption below high DRL were included in Cohort-B. Cohort-A received simple psy-
\end{abstract}

chosocial interventions and were supplied with nalmefene $18 \mathrm{mg}$ to be taken on days when they perceived a risk of drinking alcohol. Patients in Cohort-B received a simple psychosocial intervention and were treated per normal practice. Results: Of the 378 enrolled patients, 330 were included in Cohort-A and 48 in Cohort-B. For patients in Cohort-A, the mean change from screening to Week-12 in the number of heavy drinking days/month was -13.1 days/month $(95 \% \mathrm{Cl}$ -14.4 to $-11.9, p<0.0001)$. Overall, $55 \%$ of patients reduced their DRL by $\geq 2$ risk levels and $44 \%$ of patients reduced to a low DRL. The most common adverse events were nausea (18.3\%) and dizziness (17.7\%). Patients in Cohort-B maintained their lower level alcohol consumption at the 12-week follow-up. Conclusions: Patients with alcohol dependence treated in primary care with nalmefene, taken as needed, in conjunction with simple psychosocial support, significantly reduced their alcohol consumption. Treatment was well tolerated.

\section{KARGER}

(c) 2018 S. Karger AG, Basel

E-Mail karger@karger.com

www.karger.com/ear
Philippe Castera

Professeur Associé de Médecine générale De l'Université De Bordeaux Cabinet Medical

FR-33000 Bordeaux (France)

E-Mail philippe.castera@u-bordeaux.fr 


\section{Introduction}

Excessive alcohol consumption has been recognized as a direct cause of more than 30 diseases, including alcohol dependence, alcohol psychoses, alcoholic gastritis, and alcoholic liver cirrhosis and as a significant contributor to more than 60 diseases, including certain cancers, cardiovascular disease and mood and anxiety disorders [1]. It was estimated that, in 2012, alcohol was responsible for $5.9 \%$ of all global deaths and $5.1 \%$ of all Disability-Adjusted Life Years [1]. Over time, consistent unhealthy drinking may lead to the development of alcohol dependence, where an individual is physically and/or psychologically dependent upon drinking alcohol $[2,3]$. Despite the associated high level of disease burden, alcohol dependence has the lowest treatment rate among all the major mental disorders [4]. A recent study found that at least a quarter of people with persistent alcohol use disorders, who could benefit from specific treatment, do not seek treatment [5]. For many dependent patients, total abstinence is not considered an achievable aim of treatment, whereas reduction of alcohol consumption is often accepted as an alternative approach to reducing negative consequences [6].

Nalmefene (Selincro ${ }^{\circledR}$, H. Lundbeck A/S, Valby, Denmark) is an opioid system modulator licensed in Europe for the reduction of alcohol consumption in adult patients with alcohol dependence who have a high drinking risk level (DRL) without physical withdrawal symptoms and who do not require immediate detoxification [7]. A high DRL is defined as alcohol consumption $>60 \mathrm{~g} /$ day for men and $>40 \mathrm{~g} /$ day for women. For these patients, Phase III clinical trials have shown that nalmefene, given on an as-needed basis and together with psychosocial support, reduces the total amount of alcohol consumption (TAC) and number of heavy drinking days (HDDs) and improves liver function and clinical status [8-11]. The license in the European Union was based on a post hoc analysis of the subgroup of patients who were unable to reduce their alcohol consumption during the screening period (i.e., they continued to meet inclusion criteria at randomisation) [12]. As usual for randomised controlled trials, all patients in these trials had undergone a significant screening interview and this appeared to be an effective intervention for almost $40 \%$ of patients in the 2 double-blind trials $[8,9,11]$. For this reason, the label recommends that nalmefene should be initiated only in patients who continue to have a high DRL 2 weeks after initial assessment. However, this target population has not, until now, been prospectively included in a clinical trial for nalmefene in the EU. Another Japanese Phase III study using this target population has also recently been completed (NCT02364947).

The previous Phase III trials were mostly conducted in the specialist setting. Primary care, however, has been identified as being key to improving the management of alcohol dependence. Healthcare professionals working in primary care services are well placed to manage mild to moderate alcohol dependence (i.e., those not requiring withdrawal services) in terms of recognizing and diagnosing the problem, provision of interventions, and referral of difficult cases to specialized services $[13,14]$. While workplace surveys have found that some general practitioners (GPs) feel they lack the necessary skill set and tools to tackle alcohol problems in their own setting [15-18], several studies have shown that GPs can provide effective brief interventions for alcohol-related problems, and that they can play a pivotal role in referral and relapse prevention $[14,19]$.

This study aimed to evaluate the efficacy and safety of nalmefene within the licensed indication in the primary care setting where local constraints and conditions (e.g., time and patient populations) are prioritized over the optimal settings provided in controlled trials.

\section{Methods}

This was an interventional, 12-week, open-label study with nalmefene, as-needed use. The study (NCT02195817) was conducted in 43 primary care sites in 4 countries (France, Germany, Spain and UK) between the August 5, 2014 and the September 25, 2015 where it was terminated early due to difficulties in site and patient recruitment. All ongoing patients at the time of study termination could complete the study. The primary care sites were selected based on some previous experience with clinical studies. Sites should have been interested in the management of alcohol dependence, or could have had some prior experience, but the management of alcohol dependence should have not represented the main part of the GP investigators daily practice. Local and regional institutional review boards covering the participating sites approved the protocol and the trial was executed in accordance with the Declaration of Helsinki and International Conference on Harmonization Good Clinical Practice Guidelines. All patients provided written informed consent to participate in the trial.

\section{Patients}

Male and female adults (aged $\geq 18$ years) with a diagnosis of alcohol dependence according to ICD-10 criteria [3] and seeing a GP were recruited to the study. Patients could be recruited from the study sites' own patient pool, by patient self-referral (e.g., leaflets at GP sites were permitted in Germany), or referrals to the study site. Patients had to have an average alcohol consumption of at least a high DRL in the 4 weeks preceding the Screening Visit. 


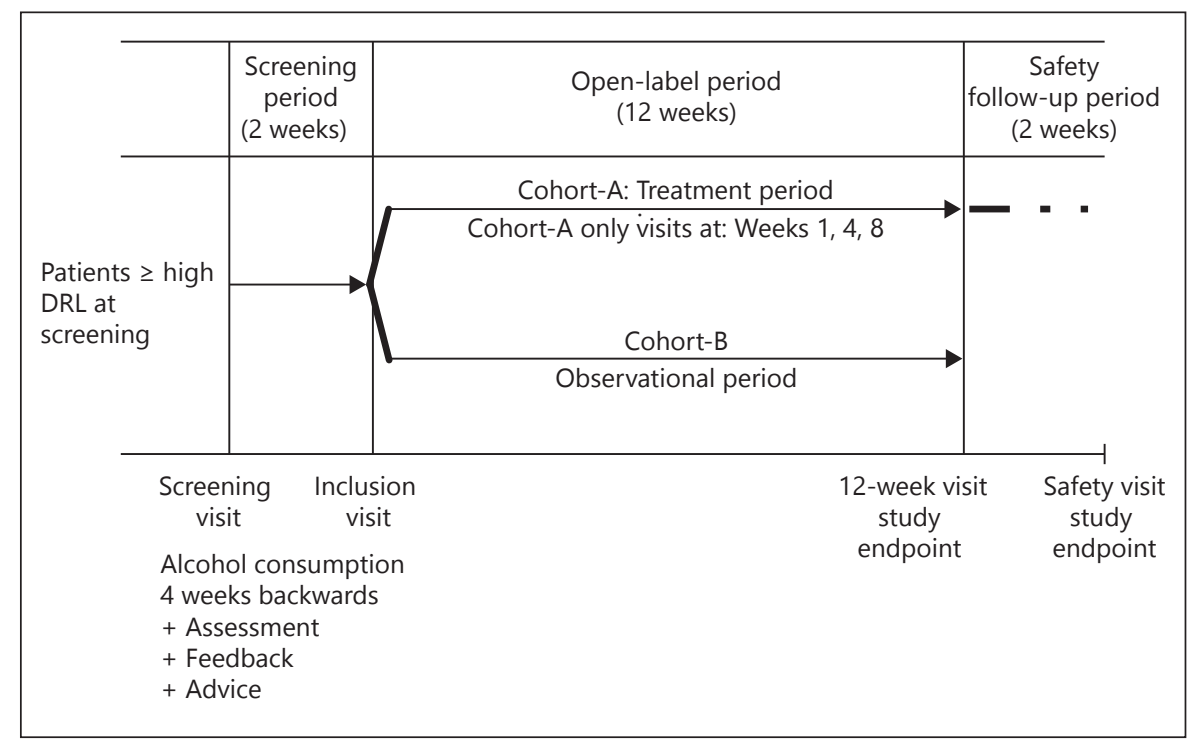

Fig. 1. Study design.

In addition, patients had to provide a stable address, be willing and able to attend appointments within the specified time windows and identify a locator person that could be contacted in case of loss of contact during the study.

Key exclusion criteria were any contradiction to the prescribing information for nalmefene (hypersensitivity to the active substance or to any of the excipients, taking opioid analgesics, current or recent opioid addiction, acute symptoms of opioid withdrawal, recent use of opioids suspected, severe hepatic or renal impairment, or a recent history of acute alcohol withdrawal syndrome), the presence of physical alcohol withdrawal symptoms requiring immediate in-patient detoxification, severe psychiatric disorders or important physical comorbidities. Patients with a recent (within past 4 weeks) or current history of participating in treatment or support programs for alcohol-use disorders or previous use of nalmefene (within past 6 months) were also excluded from this trial.

\section{Study Design}

The study was conducted in 3 parts: a screening period, an open-label treatment period and a safety follow-up period (Fig. 1). Patients' clinical status, alcohol dependence diagnosis and severity, social and family situation, alcohol consumption and treatment goal were assessed at the first visit (Screening Visit). Patients were asked to record their alcohol consumption for the next 2 weeks. At the second visit (Inclusion Visit), patients were categorized into 2 cohorts depending on their reported level of drinking over the past 2 weeks, as identified using the Timeline Follow-back (TLFB) method [20] to provide information of daily number of standard drinks consumed. Cohort-A comprised those patients who maintained at least a high DRL in the period between the Screening and Inclusion visits. Cohort-B comprised those patients who reduced their alcohol consumption below high DRL, and were therefore not eligible for treatment with nalmefene.

At the Inclusion Visit, patients in Cohort-A were supplied with nalmefene $(18 \mathrm{mg})$, and received a brief psychosocial intervention based on motivational interviewing with a focus on alcohol reduction and treatment adherence - but not a formal, manual-guided psychosocial intervention. All investigators had received training in biopsychosocial assessment, which included feedback to investigators on their assessments, assessing patient's treatment goal, providing advice to reduce alcohol drinking, and monitoring of the treatment progress. Patients were instructed to take 1 tablet each day they perceived a risk of drinking alcohol (as-needed dosing), preferably $1-2 \mathrm{~h}$ prior to anticipated time of drinking, but otherwise as soon as drinking had started. Nalmefene was dispensed at each visit and patients were supplied with enough nalmefene to allow 1 tablet per day until the next visit. For Cohort-A, the continuous psychosocial support during the subsequent 12week treatment period comprised of monitoring the patients' treatment progress (focusing on reduction of alcohol consumption) and providing guidance on the correct use of nalmefene (focusing on treatment adherence).

Patients in Cohort-B received a brief psychosocial intervention at the Inclusion Visit focused on encouraging them to maintain their reduced alcohol consumption. Thereafter patients in Cohort$\mathrm{B}$ were treated per normal clinical practice.

\section{Outcomes}

For both cohorts, assessments were performed at the Screening and Inclusion Visits and at Week 12 (study endpoint) of the openlabel treatment period. Patients in Cohort-A were also assessed at Week 1, 4 and 8 of the open-label treatment period. Drinking variables were derived from the TLFB method, and covered the period between the current visit and the previous visit.

For patients in Cohort-A, the primary endpoint was the change from the Screening Visit to Week 12 of the open-label treatment period in the number of HDDs (days/month, where 1 month is defined as 28 consecutive days). Secondary endpoints included:

- Change from Inclusion Visit to Week 12 in TAC (g/day) and Global Impression-Severity of Illness (CGI-S) scores rated on a 7 -point scale ranging from 1 (normal - not at all ill) to 7 (among the most extremely ill patients). Clinical Global Impression of Improvement (CGI-I) was assessed at Week 12 using a 7 -point scale ranging from 1 (very much improved) to 7 (very much worse). 
- Responder analyses based on drinking measures were also conducted to assess the proportion of patients who showed a downward shift between Inclusion Visit and Week 12 in: (i) from a very high DRL ( $>100 \mathrm{~g} /$ day for men and $>60 \mathrm{~g} /$ day for women) to medium DRL (40-60 g/day for men and 20-40 g/day for women) and below, or from a high DRL to low DRL (1-40 g/day for men and 1-20 g/day for women) and below (ii) a shift to a low DRL (iii) a $\geq 50 \%$ reduction in TAC (iv) a reduction of drinking to $0-4$ HDDs per month.

- Liver function variables were assessed at Screening Visit and Week 12, and included: $\gamma$-glutamyltransferase (GGT), alanine aminotransferase (ALT) and aspartate aminotransferase (AST).

In addition, the change from Screening Visit in the 36-item Short-form Health Survey version 2 (SF-36) [21] was assessed as a secondary measure for patients enrolled at the UK and French sites. Safety assessments consisted of evaluation of adverse events (AEs), clinical safety laboratory tests, vital signs and weight.

For patients in Cohort-B, changes from Inclusion Visit to Week 12 in the number of HDDs and TAC were assessed as exploratory endpoints.

\section{Statistical Analyses}

Demographic data and patient disposition for both Cohorts are presented for all enrolled patients. Safety analyses for Cohort-A were conducted on the all patients-treated set, which included all patients who recorded an intake of $\geq 1$ nalmefene. Efficacy analyses for Cohort-A were conducted in the Full Analysis Set, which included all patients with $\geq 1$ valid post-inclusion assessment of the primary efficacy variable. The primary efficacy variable (change from Screening Visit in HDDs) was analysed using a mixed model for repeated measurements, using all available data with sex, site, and time in months as factors and the number of HDDs at screening included as a covariate; number of HDDs at screening-bymonth interaction was also included in the model. Within-patient errors were modelled using an unstructured covariance structure and the estimation method was based on a restricted maximum likelihood-based approach. Subgroup analyses by country for the change from Screening Visit in the number of HDDs in Cohort-A were performed on an exploratory basis, using the same methodology as that for the total Cohort-A.

The reduction in TAC was analysed in the same way as the primary efficacy measure, CGI-S and CGI-I scores and reductions in log-transformed GGT, ALT and AST values were analysed with similar models as described above. The CGI-S score at the Inclusion Visit was included as a covariate in the model for the CGI-I. For responder endpoints, the percentage of responders with corresponding 2-sided 95\% CIs was based on normal approximation. Safety and exploratory efficacy analyses for Cohort-B were descriptive only (observed data) and included all patients. All statistical tests were 2 -sided and conducted at the $5 \%$ level of significance. The statistical software used was $S A S^{\circledR}$, Version 9.4.

\section{Sample Size Calculation}

Sample size was based on the findings of the pivotal studies [8, 9]. Based on an SD of 10.5 and a withdrawal rate of $20 \%$ at Week 12 , a sample size of 475 patients treated with nalmefene was estimated to provide a probability of about 0.85 for the half width of the 2 -sided $95 \%$ CI to be at most 1.1 HDDs per month. To account for those patients who would reduce their alcohol consumption following just the Screening Visit (18-33\% in pivotal studies) and would enter Cohort-B, we originally planned for recruitment of 635 patients.

Although the study was terminated early, 330 patients had already been assigned to Cohort-A. Assuming an SD of 10.5 and a $20 \%$ withdrawal rate at Week 12 , this provided a probability of around 0.81 for the half width of the 2 -sided $95 \%$ CI to be at most 1.32 HDDs.

\section{Results}

\section{Patient Disposition}

A total of 378 patients fulfilled the screening criteria, and were enrolled in the study, and of these 330 were unable to reduce their alcohol consumption in the 2 weeks following the Screening Visit and were enrolled in Cohort-A. For Cohort-A, the mean TAC was $111 \mathrm{~g} /$ day at the Screening Visit and remained above high DRL at the Inclusion Visit (106 g/day). Of the patients enrolled in Cohort-A, 268 (81.2\%) completed the 12-week study. Main reasons for study withdrawal were withdrawal of consent (6.4\%) and adverse events (5.5\%). Figure 2 shows the disposition of all patients throughout the study.

Forty-eight patients managed to reduce their alcohol consumption to a medium or low DRL during the 2 weeks following the Screening Visit (from a mean TAC of $84 \mathrm{~g} /$ day at screening to $31 \mathrm{~g} /$ day at the Inclusion Visit) and were enrolled in Cohort-B.

Clinical and demographic characteristics for each cohort are provided in Table 1. The majority of patients in both cohorts were Caucasian, and the mean age was 52 years in Cohort-A and 55 years in Cohort-B. Overall, 85\% of patients in each of the cohorts had concurrent medical or psychiatric disorders, and about 3 quarters (74\% in Cohort- $\mathrm{A}$ and $77 \%$ in Cohort-B) had never been treated for alcohol disorders. There was a male predominance in both cohorts, with highest proportion of males in Cohort-B.

\section{Exposure to Nalmefene (18 mg/day) in Cohort- $A$}

On average, patients in Cohort-A took a dose of nalmefene on $54 \%$ of the days. The mean percentage of days that patients were adherent (defined as days with drinking and with intake of nalmefene, no drinking and with intake of nalmefene, or no drinking and with no intake of nalmefene) was $70 \%$, with $47 \%$ of patients being adherent on $\geq 80 \%$ of the days.

\section{Cohort-A: Reduction in Alcohol Consumption During the Open-Label Period}

For patients in Cohort-A, treatment resulted in a continued reduction in the mean number of HDDs 


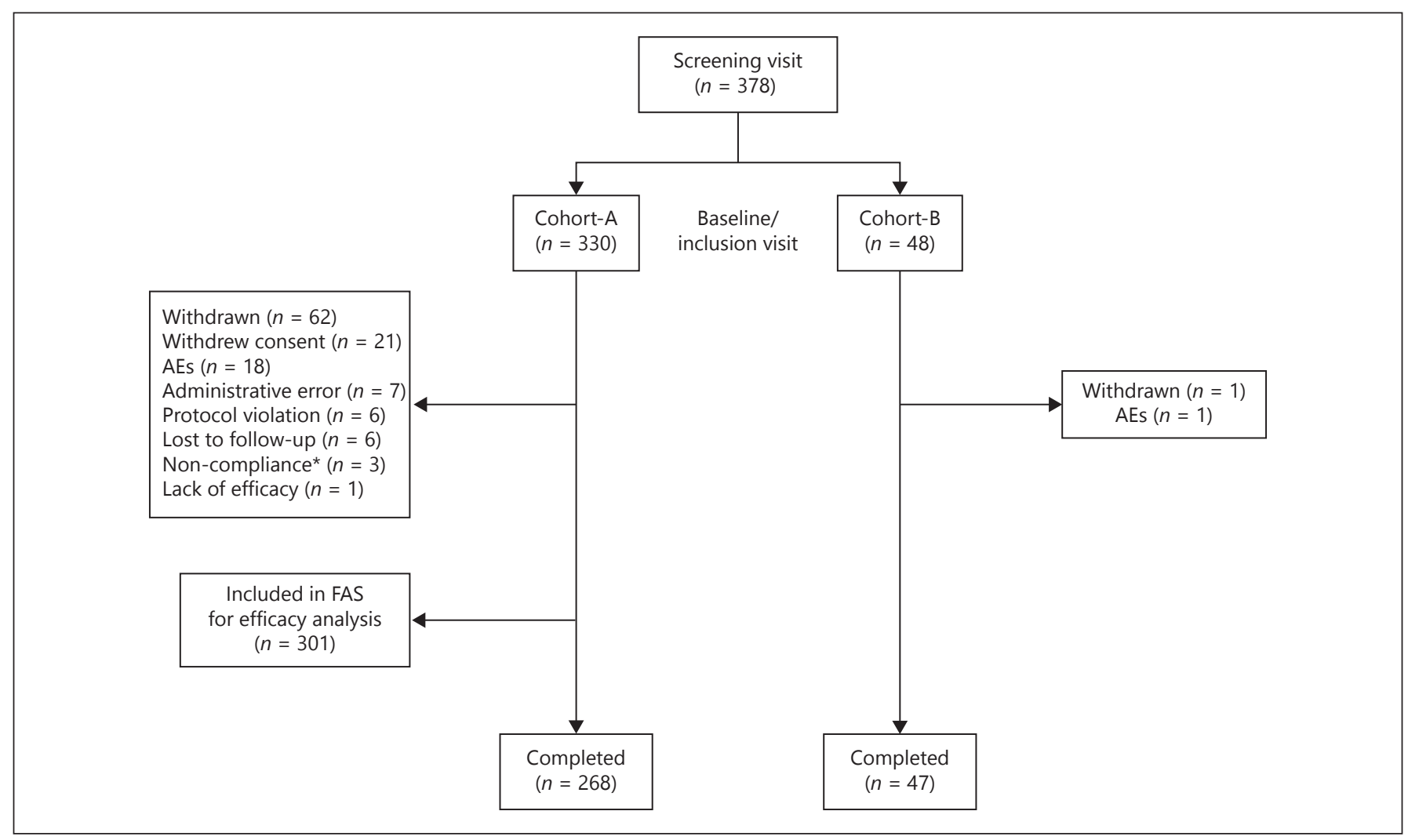

Fig. 2. Patient disposition. *Non-compliance to study medication was judged by the investigator. AEs, adverse events; FAS, full analysis set.

over time. The mean number of HDDs decreased from 24 days/month at the Screening Visit to 15 days/month at week 4 , and to 11 days/month at Week 12 . The adjusted mean change from the Screening Visit in the number of HDDs at Week 12 (primary endpoint) was -13.1 days/month (95\% CI -14.4 to -11.9 ; Fig. 3a). Likewise, there was a significant reduction in TAC; mean TAC decreased from $111 \mathrm{~g} /$ day at the Screening Visit to $67 \mathrm{~g} /$ day at Week 4, and to $50 \mathrm{~g} /$ day at Week 12. The adjusted mean change from Screening Visit in TAC at Week 12 was $-64.0 \mathrm{~g} /$ day (95\% CI -69.4 to -58.6; Fig. 3b). Patients in all countries showed reduction in their number of HDDs; however, there was some national variation in drinking levels and the subsequent reductions.

Responder analyses also supported the primary efficacy findings, with $55 \%$ of patients reducing their DRL by at least 2 risk levels and $44 \%$ of patients reducing to low DRL. Overall, $61 \%$ of patients were able to reduce their TAC by at least 50 , and $38 \%$ of patients could reduce their drinking to $0-4 \mathrm{HDDs} /$ month (Table 2).

\section{Other Efficacy Outcomes}

Reductions in alcohol consumption were reflected in continued improvements in CGI-S scores, which improved from a mean of 4.0 at the Inclusion Visit to 2.8 at Week 12. The mean CGI-I score at Week 12 was 2.5 (95\% CI 2.3-2.6). In addition, the results of liver function tests showed a benefit of treatment with nalmefene; mean levels of GGT, ALT, and AST all decreased over time from the Screening Visit to Week 12 (Table 2). For patients in the United Kingdom and France $(n=122)$, mean changes from Screening Visit to Week 12 in SF-36 scores were 7.7 points (95\% CI 4.9-10.5) for the mental component and 2.2 points (95\% CI $0.6-3.9)$ for the physical component score.

\section{Cohort-B: Alcohol Consumption During the Open-Label Period}

Patients in Cohort-B considerably reduced their alcohol consumption during the 2 weeks following the Screening Visit. During the screening period, the mean number of HDDs reduced from 23 to 5 days/month and the mean 
Table 1. Patient characteristics

\begin{tabular}{|c|c|c|}
\hline & Cohort-A & Cohort-B \\
\hline \multicolumn{3}{|l|}{ Demographics and medical history } \\
\hline Number, $n$ & 330 & 48 \\
\hline Age, years, mean (SD) & $51.98(11.25)$ & $54.83(12.40)$ \\
\hline \multicolumn{3}{|l|}{ Gender, $n(\%)$} \\
\hline Male & $216(65.5)$ & $39(81.3)$ \\
\hline Female & $114(34.5)$ & $9(18.8)$ \\
\hline \multicolumn{3}{|l|}{ Race, $n(\%)$} \\
\hline White caucasian & $328(99.4)$ & $47(97.9)$ \\
\hline Other & $2(0.6)$ & $1(2.1)$ \\
\hline \multicolumn{3}{|l|}{ BMI, mean, SD } \\
\hline Male & $27.94(5.14)$ & $28.35(4.48)$ \\
\hline Female & $26.30(5.78)$ & $24.27(5.26)$ \\
\hline Age of drinking problem onset, years, mean (SD) & $37.45(13.85)$ & $39.73(13.35)$ \\
\hline Concurrent medical and psychiatric disorders, $n(\%)$ & $279(84.5)$ & $41(85.4)$ \\
\hline Hypertension & $108(32.7)$ & $20(41.7)$ \\
\hline Depressive symptoms & $42(12.7)$ & $3(6.3)$ \\
\hline Type 2 diabetes mellitus & $35(10.6)$ & $5(10.4)$ \\
\hline Hypercholesterolemia & $33(10.0)$ & $12(25.0)$ \\
\hline Family history of alcohol problems, $n$ (\%) & $176(53.3)$ & $30(62.5)$ \\
\hline Previous treatment for alcohol disorders, $n(\%)$ & $85(25.8)$ & $11(22.9)$ \\
\hline Current smoking, $n(\%)$ & $263(79.7)$ & $43(89.6)$ \\
\hline Current nicotine use (excluding smoking), $n(\%)$ & $93(28.2)$ & $3(6.3)$ \\
\hline \multicolumn{3}{|l|}{ Screening and inclusion visit drinking variables } \\
\hline$n$ & 301 & 48 \\
\hline \multicolumn{3}{|l|}{ HDD, days/month, mean (SD) } \\
\hline Screening visit & $24.14(5.09)$ & $23.17(5.76)$ \\
\hline Inclusion visit & $23.67(5.27)$ & $5.46(0.77)$ \\
\hline \multicolumn{3}{|l|}{ TAC, g/day, mean (SD) } \\
\hline Screening visit & $111.23(53.02)$ & $83.77(36.38)$ \\
\hline Inclusion visit & $105.86(50.25)$ & $31.33(2.62)$ \\
\hline
\end{tabular}

BMI, body mass index; HDD, heavy drinking days; TAC, total alcohol consumption.

TAC reduced from 84 to $31 \mathrm{~g} /$ day. Exploratory analyses showed that patients in Cohort-B were generally able to maintain their lower level alcohol consumption and DRL for at least 12 weeks. By week 12, the mean number of HDDs was 7 days/month (increase from Inclusion Visit of $1.551 \pm 8.9$ days/month) and the mean TAC was $33 \mathrm{~g} /$ day (increase from Inclusion Visit of $1.34 \pm 20.52 \mathrm{~g} /$ day).

\section{Safety and Tolerability}

Nalmefene was generally well tolerated. A total of 19 patients (6.4\%) in Cohort A withdrew from the study due to adverse events; the most common reasons were nausea in 6 patients (2\%) and dizziness in 3 patients (1\%). One patient in Cohort B withdrew due to a TEAE (anxiety). Overall, $68 \%$ of patients in Cohort-A had 1 or more TEAEs (Table 3), most of which were mild or moderate in severity. A total of 22 patients (7\%) reported 26 treatment-emergent SAEs. SAE's occurring in $\geq 1$ patient were alcohol withdrawal syndrome, depressive symptoms, and hepatic cirrhosis (all $n=2$ ). Two patients died during the study. One 57-year-old male had hepatorenal syndrome during the screening period (i.e., before allocation to Cohort-A or Cohort-B), was hospitalized and died 4 days after the event. Another 44-year-old male patient in Cohort-A died 79 days after the first dose of nalmefene due to hepatic cirrhosis and decompensated liver cirrhosis (assessed by the investigator as possibly related to study drug) and hepatic encephalopathy (assessed as not-related to study drug). The investigator specified "long-term intake of excess alcohol" as aetiology. Other than the liver function tests described above, no clinically relevant changes were noted for clinical safety laboratory tests or 


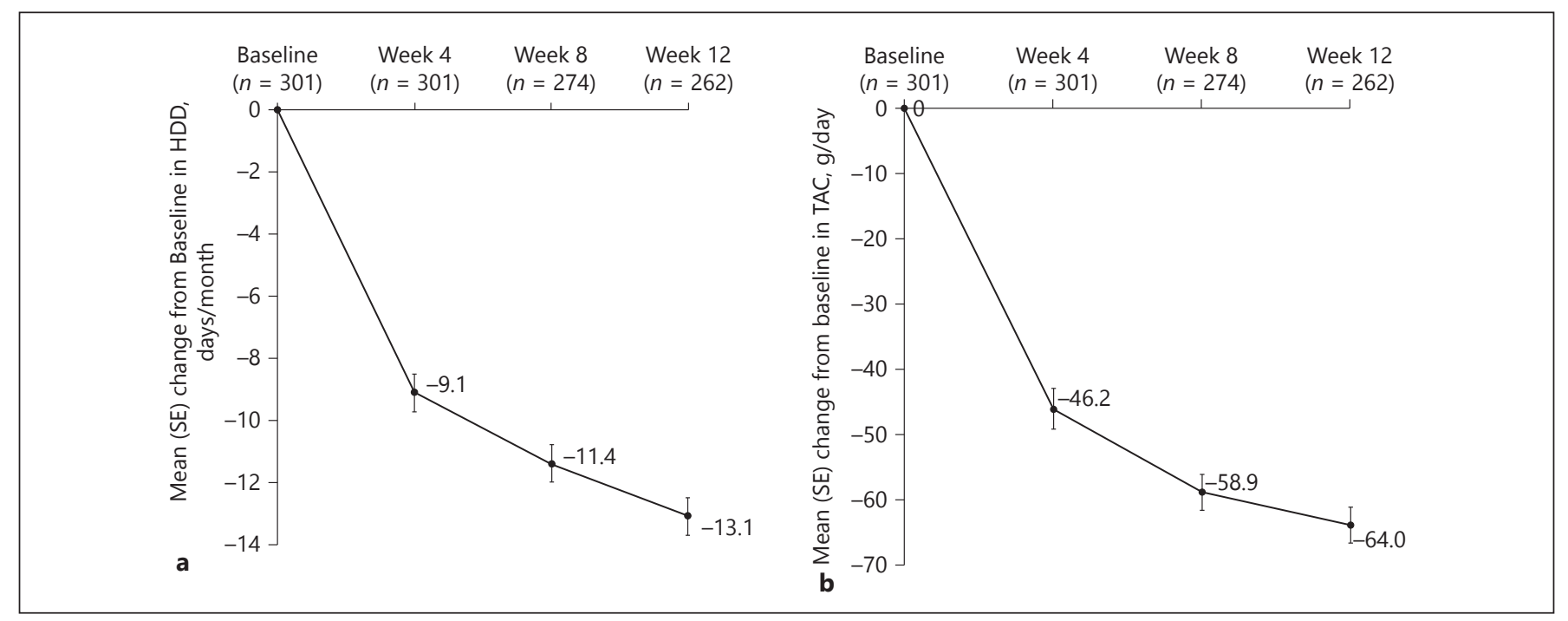

Fig. 3. Reduction in alcohol consumption in Cohort-A (a) Primary endpoint: number of heavy drinking days (HDD) per month (primary endpoint), (b) Secondary endpoint: total alcohol consumption (TAC) per day. Data show adjusted change from Screen-

vital signs. On average, patients in Cohort-A had a mean weight loss of $0.4 \mathrm{~kg}$ versus Inclusion Visit; 9 patients (3\%) had a potentially clinically relevant weight decrease and $6(2 \%)$ had a potentially clinically relevant weight increase.

The only TEAE to occur in $>5 \%$ of patients in Cohort$\mathrm{B}$ was nasopharyngitis $(n=4 ; 8.3 \%)$. One patient in Cohort-B withdrew due to an $\mathrm{AE}$ (anxiety) and 1 patient had an SAE of bronchospasm.

\section{Discussion}

This was the first study of nalmefene for alcohol dependence in the primary care setting and showed that 3 months of as-needed treatment in conjunction with simple psychosocial support significantly reduced alcohol consumption as assessed by a reduction in HDDs per month and daily TAC. As-needed treatment was generally well tolerated and comprised adverse events consistent with the known safety profile [8-10]; no new safety concerns were identified in this study.

The magnitude of the reductions in alcohol consumption seen in this study (approximately 54\% reduction in HDDs per month and 58\% reduction in daily TAC) are in line with that seen with active treatment in the pivotal placebo-controlled studies $[8,9]$. Another recently completed Japanese Phase III study of nalmefene (which, ing Visit based on a MMRM model with sex, site, and time in months as fixed effects and baseline values as covariates and baseline by time interaction.

like this study, was conducted in alcohol-dependent patients who could not reduce their alcohol intake after a brief intervention) also showed a significantly reduction in the number of HDD and TAC versus placebo [22]. Also like the pivotal studies, the treatment effects on HDD and TAC were observed as early as week 4 and continued to reduce throughout the treatment period. The clinical relevance of these reductions is highlighted by the fact that $55 \%$ of patients could reduce their DRL by at least 2 levels and relevant proportions of patients met other definitions of having a good treatment response. Since there is a dose-dependent relationship between alcohol intake and health-related harms [23], reducing alcohol consumption to lower risk levels would be expected to be associated with health benefits such as reductions in blood pressure in hypertensive subjects [24] and remission of alcohol-related depressive symptoms [25]. The proportion of patients who could reduce their alcohol consumption to a "low" DRL was smaller than the proportion of patients who reduced their DRL by at least 2 levels (43.9 vs. 55.3\%). This discrepancy was largely driven by patients in the United Kingdom whose drinking levels were higher than the other countries; for example, the mean daily TAC at screening was $135 \mathrm{~g} /$ day in the United Kingdom versus 96 g/day in Germany. Thus, while patients in the United Kingdom reduced their TAC by an average of $49 \%$, they were still drinking at medium or high DRL. 
Table 2. Secondary efficacy outcomes in cohort-A

\begin{tabular}{|c|c|}
\hline Efficacy parameter & Statistic \\
\hline $\begin{array}{l}\text { Proportion of patients achieving a reduction of at least } 2 \text { DRLs } \\
\text { from inclusion visit to week } 12, \%(95 \% \mathrm{CI})\end{array}$ & $\begin{array}{l}(n=262) \\
55.3(49.3-61.2)\end{array}$ \\
\hline Proportion of patients achieving at least a low DRL at week $12, n(\% ; 95 \% \mathrm{CI})$ & $\begin{array}{l}(n=262) \\
43.9(38.0-49.9)\end{array}$ \\
\hline $\begin{array}{l}\text { Proportion of patients achieving at least a } 50 \% \text { reduction in daily TAC } \\
\text { from inclusion visit to week } 12, n(\% ; 95 \% \mathrm{CI})\end{array}$ & $\begin{array}{l}(n=262) \\
61.1(55.0-66.8)\end{array}$ \\
\hline $\begin{array}{l}\text { Proportion of patients achieving a reduction of drinking to } \\
0-4 \text { HDDs/month, } n(\% ; 95 \% \mathrm{CI})\end{array}$ & $\begin{array}{l}(n=262) \\
37.8(32.1-43.8)\end{array}$ \\
\hline $\begin{array}{l}\text { CGI-S } \\
\text { Inclusion Visit score (absolute value), mean (SD) } \\
\text { change from inclusion visit to week } 12 \text { in CGI-S score, mean (SE) }\end{array}$ & $\begin{array}{l}(n=265) \\
4.01(0.95) \\
-1.2(0.1)\end{array}$ \\
\hline CGI-I score at week 12, mean (SE) & $\begin{array}{l}(n=265) \\
2.5(0.1)\end{array}$ \\
\hline $\begin{array}{l}\text { Liver function tests } \\
\text { Change in GGT, geometric mean (95\% CI) } \\
\text { Screening visit } \\
\text { Geometric mean ratio at week } 12 \text { (MMRM)* }\end{array}$ & $\begin{array}{l}69.55 \\
0.87(0.80-0.95)\end{array}$ \\
\hline $\begin{array}{l}A L T \\
\text { Screening visit } \\
\text { Geometric mean ratio at week } 12(\mathrm{MMRM})^{*}\end{array}$ & $\begin{array}{l}29.22 \\
0.90(0.84-0.97)\end{array}$ \\
\hline $\begin{array}{l}\text { AST } \\
\text { Screening visit } \\
\text { Geometric mean ratio at week } 12(\mathrm{MMRM})^{*}\end{array}$ & $\begin{array}{l}32.17 \\
0.89(0.83-0.95)\end{array}$ \\
\hline
\end{tabular}

* A value of 1.0 reflects no change.

DRLs, drinking risk levels; TAC, total amount of alcohol consumption; HDD, heavy drinking day; CGI-S, Global Impression-Severity of Illness; CGI-I, Clinical Global Impression of Improvement; GGT, $\gamma$-glutamyltransferase; ALT, alanine aminotransferase; AST, aspartate aminotransferase.

Table 3. Summary of adverse events

\begin{tabular}{lcc}
\hline & $\begin{array}{l}\text { Cohort-A } \\
(n=330)\end{array}$ & $\begin{array}{l}\text { Cohort-B } \\
(n=48)\end{array}$ \\
\hline$\geq 1$ TEAE, $n(\%)$ & $211(63.9)$ & $16(33.3)$ \\
SAEs, $n(\%)$ & $27(8.2)$ & $1(2.1)$ \\
AE leading to withdrawal, $n(\%)$ & $21(6.4)$ & $1(2.1)$ \\
Death & $1(0.3)$ & - \\
AEs occurring in $>5 \%$ of & & \\
$\quad$ patients in cohort-A & $57(18.3)$ & $1(2.1)$ \\
$\quad$ Nausea & $55(17.7)$ & - \\
$\quad$ Dizziness & $33(10.6)$ & - \\
Insomnia & $24(7.7)$ & $1(2.1)$ \\
Headache & $19(6.1)$ & - \\
$\quad$ Vomiting & \multicolumn{2}{c}{} \\
\hline
\end{tabular}

The clinical utility of nalmefene treatment is supported by the overall clinical global impression of patients at the end of the study. A mean CGI-I score of 2.5 indicates a score between "much improved" and "improved," and this finding highlights the fact that the GPs could discern a difference in the clinical condition of their patients after just 12 weeks of treatment. Patients in the United Kingdom and France showed a mean change of 7.7 points in the SF-36 mental component score, which is well above the minimally clinically relevant difference of 3.2 points [26] and indicates a highly clinically relevant improvement in mental health. In addition, laboratory tests showed that treatment with nalmefene resulted in a reduction in levels of GGT, ALT and AST, thus providing objective verification of efficacy data gained from the TLFB method of patient self-reported drinking. 
Importantly, this study suggests that the level of psychosocial support required to support candidate patients for nalmefene can easily be implemented in primary care. In this study, the psychosocial support was based on motivational interviewing focused on treatment adherence and reducing alcohol consumption - no specific manualguided interventions were adopted. About $12 \%$ of patients significantly reduced their alcohol consumption during the screening period and therefore did not require pharmacotherapy. Of note, patients in Cohort-B had a similar number of HDDs per month compared to patients in Cohort-A, but a lower daily TAC at Screening. Patients in this group reduced their alcohol consumption to moderate-low DRLs within the 2 weeks between the Screening and Inclusion visits, and maintained this level for at least 12 weeks. This supports the premise that a significant number of patients who make the decision to seek help for their excessive drinking are able to reduce their alcohol consumption following a brief interventionlike approach provided in primary care [27].

It has been proposed that by giving people the option of starting treatment with reduction and defining their own staged treatment goals, there is the potential to encourage more dependent drinkers to seek professional help, since many patients do not find abstinence an acceptable goal [6,28-30]. Indeed, around 3 quarters of patients enrolled in this study were new to treatment for alcohol dependence. Within this context, our study supports the clinical utility of nalmefene for those with a goal of reduction requiring the use of pharmacotherapy to help achieve their goal. The as-needed approach has been suggested to not only empower patients in making daily treatment decisions but also increase awareness of the amount of alcohol consumed and drinking patterns, thus facilitating the identification of at-risk situations, which is an integral part of disease management [30]. Patients appeared to understand and accept the concept of as needed use. As in the pivotal studies, patients in Cohort-A took nalmefene on about half of the study days. While overall adherence was good (70\%), fewer patients were adherent on $\geq 80 \%$ of days than in the pivotal studies ( 47 vs. $68 \%$ ) [31].

While randomized controlled studies are considered the "gold standard" design, the efficacy of nalmefene has already been evaluated under such conditions [8-11], and open-label studies such as this are considered to provide a better opportunities to mimic routine practice in primary care. Since this study was designed to test the currently recommended treatment paradigm for nalmefene, it only recruited patients with at least a high DRL, and we did not study the utility of nalmefene in patients with a medium DRL (which were included in the pivotal studies [8-10]). Of note, patient demographic and clinical characteristics in terms of age, age at onset, family history and prior treatment for alcohol dependency were fairly similar to the pivotal trials $[8,9]$, but older and with an older age of onset than patients recruited to the recent Spanish Phase IV study, which included a relatively high proportion of patients at low (45.4\%) and medium (16.4\%) DRL [32]. The open-label design also allowed us to explore the alcohol consumption of the patients who benefit from the psychosocial intervention at the Screening Visit (i.e., Cohort-B) under conditions that to a greater degree resemble real-life than was possible in the pivotal Phase III studies. At the Screening Visit, patient demographic and clinical characteristics for Cohort-A and Cohort-B were similar, and although not formally analysed, there did not appear to be any obvious differences between the 2 subsamples.

This study suffers from several inherent limitations, including the fact that the study was early terminated due to slow recruitment in some countries, which resulted in fewer patients than originally planned. Nevertheless, the study did include sufficient patient numbers for analysis and significant reductions in alcohol consumption were clearly demonstrated. As in most other trials in patients with alcohol abuse or dependency, in the absence of a generally agreed objective measure of alcohol consumption, the findings relied on patient self-report. Although the study was limited to 12 weeks of open-label treatment, previous experience in the 6-month studies with nalmefene showed that most of the reduction in alcohol consumption occurs within the first month and that any changes from 3 to 6 months were small $[8,9,11]$. A similar pattern of efficacy was also noted in the long-term study where patients treated with nalmefene and psychosocial support maintained a reduction in alcohol consumption for up to 1 year [10].

\section{Conclusion}

Treatment with nalmefene, taken as needed, in conjunction with simple psychosocial support significantly reduced alcohol consumption in patients with alcohol dependence treated in primary care and was generally well tolerated. Offering treatment with nalmefene in conjunction with psychosocial support can be said to provide the next step for alcohol dependent patients who were unable to reduce their alcohol consumption within 2 weeks of a full assessment. 


\section{Acknowledgement}

This work was supported by H. Lundbeck A/S. The authors thank all the investigators and patients who participated in this trial and would like to acknowledge the editorial assistance of Anita Chadha-Patel (ACP Clinical Communications Ltd, funded by H. Lundbeck A/S) who assisted with table preparation, referencing and final styling under the full direction from authors.

\section{Disclosure Statements}

P.C., E.S., J.G., and C.B. were all investigators in the present study, and they or their institutions received funding to participate. M.B.S., D.Z., and D.M. are employed by H. Lundbeck A/S, and B.S.B. was employed by H. Lundbeck A/S at the time of the study.

\section{Funding Source}

This work was supported by H. Lundbeck A/S.

\section{Authors Contribution}

P.C., E.S., J.G., and C.B. acted as principal investigators for sites in their countries and were involved in the study design, data acquisition, manuscript writing and review and final approval of the manuscript. M.B.S. and D.Z. were involved in the analysis of data, manuscript review and critique and final approval of the manuscript. B.S.B. and D.M. were involved in the study design, data acquisition, manuscript writing and review and final approval of the manuscript.

\section{References}

1 WHO: Global Status Report on Alcohol \& Health, 2014

2 American Psychiatric Association. Diagnostic and Statistical Manual of Mental Disorders (ed 4). Washington, American Psychiatric Association, 2000.

3 WHO. International Statistical Classification of Diseases and Related Health Problems. 10th Revision (ICD-10). Geneva, World Health Organization, 1992.

4 Wittchen HU, Jacobi F, Rehm J, Gustavsson A, Svensson M, Jonsson B, et al: The size and burden of mental disorders and other disorders of the brain in Europe 2010. Eur Neuropsychopharmacol 2011;21:655-679.

5 Tuithof M, Ten Have M, van den Brink W, Vollebergh W, de Graaf R: Treatment seeking for alcohol use disorders: Treatment gap or adequate self-selection? Eur Addict Res 2016; 22:277-285.

6 Ambrogne JA: Reduced-risk drinking as a treatment goal: what clinicians need to know. J Subst Abuse Treat 2002;22:45-53.

7 Selincro: Summary of product characteristics. Valby, Denmark. H. Lundbeck A/S; 2016.

8 Gual A, He Y, Torup L, van den Brink W, Mann K; ESENSE 2 Study Group: A randomised, double-blind, placebo-controlled, efficacy study of nalmefene, as-needed use, in patients with alcohol dependence. Eur Neuropsychopharmacol 2013;23: 1432 1442.

9 Mann K, Bladstrom A, Torup L, Gual A, van den Brink W: Extending the treatment options in alcohol dependence: a randomized controlled study of as-needed nalmefene. Biol Psychiatry 2013;73:706-713.

10 van den Brink W, Sorensen P, Torup L, Mann K, Gual A; SENSE Study Group: Long-term efficacy, tolerability and safety of nalmefene as-needed in patients with alcohol dependence: a 1-year, randomised controlled study. J Psychopharmacol 2014;28:733-744.
11 van den Brink W, Aubin HJ, Bladstrom A, Torup L, Gual A, Mann K: Efficacy of asneeded nalmefene in alcohol-dependent patients with at least a high drinking risk level: results from a subgroup analysis of two randomized controlled 6-month studies. Alcohol Alcohol 2013;48:570-578.

12 European Medicines Agency (EMA) Committee for Medicinal Products for Human Use. London, Assessment report, EMA, 2012.

13 Rehm J, Allamani A, Della Vedova R, Elekes Z, Jakubczyk A, Landsmane I, et al: General practitioners recognizing alcohol dependence: a large cross-sectional study in 6 European countries. Ann Fam Med 2015;13:28-32.

14 Kaner EF, Beyer F, Dickinson HO, Pienaar E, Campbell F, Schlesinger C, et al: Effectiveness of brief alcohol interventions in primary care populations. Cochrane Database Syst Rev 2007;CD004148.

15 Wilson GB, Lock CA, Heather N, Cassidy P, Christie MM, Kaner EF: Intervention against excessive alcohol consumption in primary health care: a survey of GPs' attitudes and practices in England 10 years on. Alcohol Alcohol 2011;46:570-577.

16 Rehm J, Prieto JA, Beier M, Duhot D, Rossi A, Schulte B, et al: The role of alcohol in the management of hypertension in patients in European primary health care practices - a survey in the largest European Union countries. BMC Fam Pract 2016;17:130.

17 Anderson P, Wojnar M, Jakubczyk A, Gual A, Reynolds J, Segura L, et al: Managing alcohol problems in general practice in Europe: results from the European ODHIN survey of general practitioners. Alcohol Alcohol 2014; 49:531-539.

18 Aira M, Kauhanen J, Larivaara P, Rautio P: Factors influencing inquiry about patients' alcohol consumption by primary health care physicians: qualitative semi-structured interview study. Fam Pract 2003;20:270-275.
19 Hutchings D, Cassidy P, Dallolio E, Pearson $P$, Heather N, Kaner E: Implementing screening and brief alcohol interventions in primary care: views from both sides of the consultation. Primary Health Care Res Development 2006;7:221-229.

20 Sobell LC, Sobell MB: Timeline follow-back: A technique for assessing self-reported ethanol consumption; in Litten RZ, Allen JP (eds): Measuring Alcohol Consumption: Psychosocial and Biological Methods. Totowa, Humana Press, 1992, pp 41-72.

21 Ware JE, Donald SC: The MOS 36-item shortform health survey (SF-36). 1. Conceptual framework and item selection. Medical Care 1992;30:473-483.

22 Miyata $H$, Nakamura I, Takahashi M, Murai Y, Tsuneyoshi K, Meulien D, et al. A Longterm Extension Study for the Phase 3 Study of Nalmefene in Patients With Alcohol Dependence in Japan [Abstract]. 19th Congress of the International Society for Biomedical Research on Alcoholism (ISBRA 2018) Kyoto, Japan September, 2018.

23 Rehm J, Zatonksi W, Taylor B, Anderson P: Epidemiology and alcohol policy in Europe. Addiction 2011;106(suppl 1):11-19.

24 Xin X, He J, Frontini MG, Ogden LG, Motsamai OI, Whelton PK: Effects of alcohol reduction on blood pressure: a meta-analysis of randomized controlled trials. Hypertension 2001;38:1112-1117.

25 Brown SA, Schuckit MA: Changes in depression among abstinent alcoholics. J Stud Alcohol 1988;49:412-417.

26 Maruish ME (ed): User's manual for the SF36v2 Health Survey (3 ed). Lincoln, QualityMetric Incorporated, 2011.

27 Beich A, Thorsen T, Rollnick S: Screening in brief intervention trials targeting excessive drinkers in general practice: systematic review and meta-analysis. BMJ 2003;327:536542 
28 Gastfriend DR, Garbutt JC, Pettinati HM, Forman RF: Reduction in heavy drinking as a treatment outcome in alcohol dependence. J Subst Abuse Treat 2007;33:71-80.

29 Heather N, Adamson SJ, Raistrick D, Slegg GP: Initial preference for drinking goal in the treatment of alcohol problems: I. Baseline differences between abstinence and non-abstinence groups. Alcohol Alcohol 2010;45:128135.
30 Luquiens A, Aubin HJ: Patient preferences and perspectives regarding reducing alcohol consumption: role of nalmefene. Patient Prefer Adherence 2014; 8:1347-52.
31 Sinclair J, Chick J, Sorensen P, Kiefer F, Batel P, Gual A: Can alcohol dependent patients adhere to an "as-needed" medication regimen? Eur Addict Res 2014;20:209-217.

32 Barrio Giménez P, Ortega L, Guardia J, Roncero C, Yuguero L, Gual A: A comparison between phase-III trials and a phase-IV study of nalmefene in alcohol use disorder patients. Is there a difference? Adicciones. 2018;0:1046. 